\title{
Optical packet routing in IP-over-WDM networks deploying two-level optical labeling
}

\author{
Ton Koonen (1), Geert Morthier (2), Jean Jennen (3), Huug de Waardt (4), \\ Piet Demeester (5)
}

(1) COBRA Research Institute, Eindhoven University of Technology, The Netherlands, a.m.j.koonen@tue.nl

(2) IMEC, University of Ghent, Belgium, Morthier@intec.rug.ac.be

(3) Bell Laboratories, Lucent Technologies Nederland, Huizen, The Netherlands, jeanjennen@lucent.com

(4) COBRA Research Institute, Eindhoven University of Technology, The Netherlands, h.d.waardt@tue.nl

(5) IMEC, University of Ghent, Belgium, Piet.Demeester@intec.rug.ac.be

\begin{abstract}
Assigning a wavelength label as well as a label in a DPSK modulation format orthogonal to the data payload significantly increases the forwarding and routing capabilities of optical packet routers in IP-over-WDM networks.
\end{abstract}

\begin{abstract}
Introduction
Packet-based data traffic is growing rapidly in today's telecommunication networks, and has overtaken circuitswitched traffic in many of them. Carrying IP packets directly over WDM channels avoiding SDH or ATM as the intermediate layers is considered to become the preferred approach towards more efficient data transport networks. Direct IP-over-WDM transport is supported by the MP $\lambda \mathrm{S}$ protocol $/ 1 /$, in which wavelength-switched channels are provisioned similar to MPLS label-switched paths. In the near future, optical routing of IP packets needs to operate at multi-wavelength Tbit/s line rates. As the majority of IP packets is relatively small (50\% is less than 522 bytes), low latency forwarding and routing techniques are required. Optical Label Switching (OLS) performs these techniques directly in the optical layer, by encapsulating IP packets with an optical label at the ingress router, and swapping the label in forwarding at the core routers $/ 2 /$. Next to labeling with a wavelength, additional label information can be attached by time-multiplexing with the payload, or by using a subcarrier $/ 2 /, / 3 /$. In this paper, it is proposed to assign a next-level label by modulating it orthogonally to the payload data. Such a two-level labeling allows more comprehensive network architectures requiring multiple addressing levels for e.g. supporting Quality-of-Service and advanced routing and traffic engineering.
\end{abstract}

\section{Two-level optical packet labeling}

In addition to the wavelength label of a packet, next-level label information can be put on the optical carrier wave in a modulation format which is orthogonal to that for the data payload. E.g., the label information is DPSK modulated on the phase, and the data are modulated on the amplitude of the carrier. Alternatively, the orthogonal label modulation format can be FSK on the optical frequency of the carrier. As illustrated in Fig. 1, in the edge nodes both the wavelength label and the DPSK (or FSK) label are set and assigned to packets (or bursts of packets) from the underlying access or metro network. In the network's core nodes, the wavelength and/or the orthogonally modulated label are used for routing the packets, and can be swapped. The orthogonal channel can also be used as an embedded control channel.

As the payload does not leave the optical domain, an entirely optical end-to-end connection path can be realised. Therefore both connectionless and connection-oriented networking can be supported, with flexible bandwidth management. The use of stacked optical labels (wavelength and special modulation format) for packet switching is completely different from the approaches studied up to now within IETF, ITU and OIF. It is envisaged that this approach has a great potential for enlarging the routing capabilities in IP-over-WDM networks.

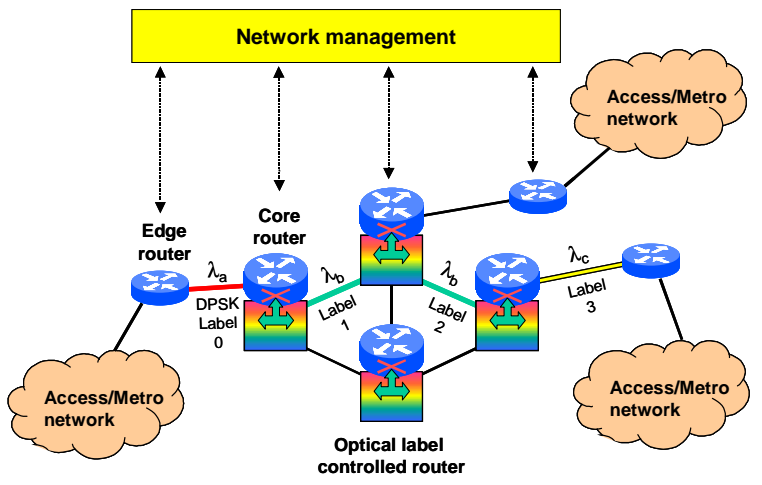

Fig. 1 Label swapping in IP-over-WDM networks

\section{Optical edge router}

The transmitter part of an edge router which assigns the two-level optical label to packets is shown in Fig. 2. After processing the incoming IP packets from the underlying access or metro network, a fast tunable laser diode followed by an external chirp-free amplitude modulator is used to carry the packets at $10 \mathrm{Gbit} / \mathrm{s}$ line rate at the appropriate wavelength in the $1.5 \mu \mathrm{m}$ region.

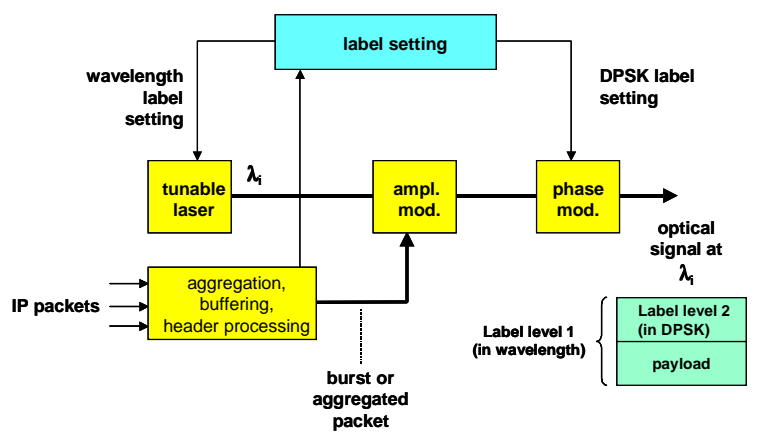

Fig. 2 Edge router with stacked optical labeling 
Tuning time of the laser is less than 500 ns over 40 ITU channels at $100 \mathrm{GHz}$ spacing, which corresponds to less than 625 bytes of guard time to be kept between IP packet bursts. The DPSK label information at rate $1 / T=155 \mathrm{Mbit} / \mathrm{s}$ is impressed by the subsequent phase modulator. Scrambling the $10 \mathrm{Gbit} / \mathrm{s}$ payload data with a $16^{\text {th }}$ order polynom (cf. ITU-T Rec. G.709) provides sufficient bit transitions to be DPSK modulated. To enable adequate DPSK demodulation, the linewidth $\delta v$ of the amplitude-modulated laser signal should meet $\delta \omega \cdot T \ll \pi$, i.e. $\delta v=\delta \omega / 2 \pi \ll 78 \mathrm{MHz}$. The DPSK modulation induces spectral broadening by $\Delta \omega \approx 2 \pi / T$, causing a jitter $\Delta \tau=D \cdot L \cdot \Delta \omega \cdot \lambda^{2} /(2 \pi c) \approx 2.5$ ps over a length $L=100 \mathrm{~km}$ of standard single-mode fibre with $D=20 \mathrm{ps} / \mathrm{nm} \cdot \mathrm{km}$, which is negligible w.r.t. the payload bit period of 100 ps.

\section{Two-level optical label swapping}

The approach for swapping the labels is shown in Fig. 3. Wavelength swapping is accomplished by using a fast tunable laser and a Mach Zehnder Interferometer (MZI) equipped with Semiconductor Optical Amplifiers (SOAs). By cross-phase modulation in the SOAs, the payload intensity modulation is transferred from the incoming wavelength to the outgoing wavelength set by the tunable laser. The phase modulation of the incoming signal, however, is stripped in the MZI, and can subsequently be replaced by phase modulation of the output signal. Thus the orthogonal DPSK-format label information can be swapped. A similar approach is applicable when an FSKformat label is used. Besides swapping the DPSK label, the wavelength converter also amplifies and reshapes the payload signal. By integrating the wavelength converter together with the phase modulator on a single InP photonic chip, a highly compact two-level label swapper is obtained.

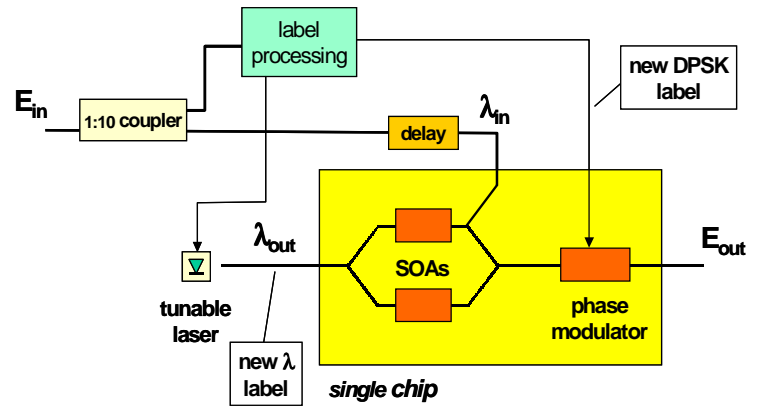

Fig. 3 Integrated two-level optical label swapper

\section{Optical label-controlled router}

Deploying the stacked-optical labels concept, a labelcontrolled router can be built as shown in Fig. 4. After demultiplexing the signals on each input fibre according to their wavelength labels, the orthogonal DPSK label is read. Each wavelength-DPSK label pair is rewritten in a new label pair according to the routing table. An arrayed waveguide grating router (AWGR) guides the pertaining packet (burst) to the appropriate output port. By using an AWGR in combination with fast widely tunable lasers for the routing, a complex and/or slow space switch is avoided. Variable delay lines are deployed to resolve contention of packets which are transposed to the same wavelength on the same output port. The delay times can be stepwise adjusted in 8 steps, by using 4 cascaded cross-bar optical switches and 3 fibre delay lines with length ratio 4:2:1. Local adding and dropping of packets to the underlying electrical IP router is accomplished by setting their wavelength appropriately.

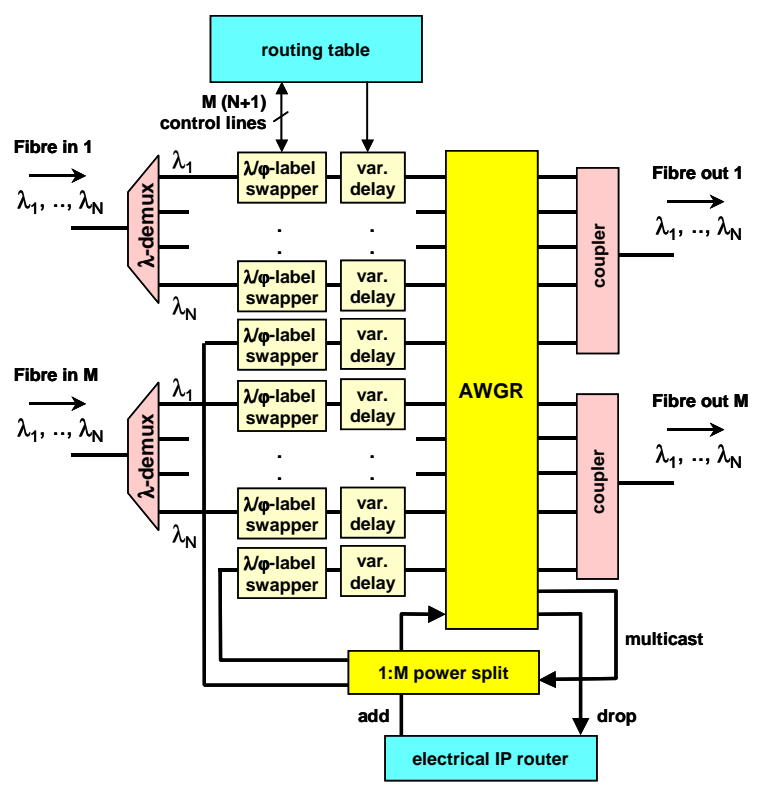

Fig. 4 Optical label-controlled router incl. multicasting

Multicasting packets is required for implementing services such as video distribution and video conferencing, and for realising virtual private networks. The multicasting function is obtained by feeding packets from a dedicated multicast output port of the AWGR back to the input ports appropriately selected by activating the tunable lasers, followed by re-labeling and routing.

Using a similar set-up, a label-controlled add/drop node including drop-and-continue function can be realised.

\section{Conclusion}

Two-level optical labeling of IP packets significantly enhances the forwarding and routing capabilities in IPover-WDM networks. Applying both a wavelength label and a label in an orthogonal modulation format allows realisation of label-controlled optical routers which significantly increase the throughput of these networks by supporting end-to-end transparent optical paths.

\section{Acknowledgement}

This work is performed in the IST STOLAS (Switching Technologies for Optically Labeled Signals) project. The other partners in the project are gratefully acknowledged for their contributions, as well as the European Commission for partly funding it.

\section{References}

/1/ N. Ghani, 'Lambda-labeling: A framework for IPover-WDM using MPLS', Optical Networks Magazine, pp. 45-58, April 2000

/2/ D.J. Blumenthal et al., "All-optical label swapping networks and technologies", IEEE J. of Lightwave Technol., Dec. 2000, pp. 2058-2075

13/ Y. Horiuchi et al., "TDM/SCM label encoding and extraction technique for lightpath control and trail monitoring", Proc. of OFC 2001, Mar. 17-23, 2001, paper ThG3 